\title{
Fixed point Theorems for Cyclic-Ćirić-Reich-Rus contraction mapping in quasi-partial b-metric spaces
}

\author{
L. N. Mishra, V. N. Mishra, P. Gautam, K. Negi
}

\begin{abstract}
In this paper, Ćirić-Reich-Rus cyclic contraction mapping is defined in the setting of quasi-partial b-metric spaces and fixed point results are proved. Some examples are given to validate our results.
\end{abstract}

Keywords: Quasi-Partial b-Metric space, Fixed point theorems, $q p_{b}$ Ćirić-Reich-Rus Cyclic Contraction mapping.

\section{Introduction}

The French mathematician Maurice Frechet initiated the study of metric spaces [1] in 1905. In 1989, Bakhtin [2] introduced the concept of b-metric space and gave the contraction mapping which was the generalization of the Banach Contraction Principle. In 1993, Czerwick [3] extended this concept of b-metric spaces whereas Shukla introduced partial $b$ metric [4] in 2014. The concept of Partial-metric spaces was introduced by Matthews [5] in 1994 as a generalization of standard metric spaces by replacing the condition $d(x, x)=0$ with the condition $d(x, x) \leq d(x, y)$, for all $x, y$. As a further generalization for quasi-metric spaces and partial-metric spaces, Karapinar [6] introduced the notion of quasi-partial metric space and discussed the existence of fixed points of self-mappings $T$ on quasi-partial metric spaces. Gupta and Gautam [7] further, generalized quasi-partial metric spaces to the class of quasi-partial b-metric spaces and proved some fixed point results ([8],[9]) in the setting of quasi-partial metric space.

In 1974, Ćirić [10]extend quasi-contraction mappings and stated some fixed point results in which it has shown that the condition of quasi-contractivity implies all conclusions

Manuscript received November 29, 2019; accepted Match 23, 2020.

L. N. Mishra is with the School of Advanced Sciences, Vellore Institute of Technology (VIT) University, Vellore, India; V. N. Mishra is with the Indira Gandhi National Tribal University Amarkantak, Madhya Pradesh, India; P. Gautam is with the Kamala Nehru College, University of Delhi,New Delhi, India; K. Negi is Research Scholar,New Delhi, India.

*Corresponding author 
of Banach's contraction principle:

Let $(X, d)$ be a metric space. A mapping $T: X \rightarrow X$ is said to be a quasi-contraction mapping if there exists $\beta \in[0,1)$ such that

$$
d(T x, T y) \leq M(x, y)
$$

for all $x, y \in X$, where

$$
M(x, y)=\max \{d(x, y), d(x, T x), d(y, T y), d(x, T y), d(y, T x)\} .
$$

Reich [11] generalized concepts of Kannan and Banach by introducing the following mapping:

A mapping $T: X \rightarrow X$ is said to be a Reich-contraction mapping if there are $\alpha, \beta, \gamma \in[0,1)$ such that $\alpha+\beta+\gamma<1$ and

$$
d(T x, T y) \leq \alpha d(x, T x)+\beta d(y, T y)+\gamma d(x, y)
$$

for all $x, y \in X$.

we also review the concept of cyclic mapping as follows:

Let $\mathrm{A}$ and $\mathrm{B}$ be non-empty subsets of a metric space $(X, d), T: A \cup B \rightarrow A \cup B$ is called cyclic if $T(A) \subset B$ and $T(B) \subset A$.

In 2016, Fan [12] proved fixed point theorems for some special cyclic mappings satisfying Banach contraction condition in the setting of a quasi-partial b-metric space as follows: Let $A$ and $B$ be nonempty subsets of a quasi-partial b-metric space $\left(X, q p_{b}\right)$. A cyclic mapping $T: A \cup B \rightarrow A \cup B$ is said to be a $q p_{b}$-cyclic-Banach contraction mapping if there exists $\alpha \in[0,1)$ such that if $s \geq 1, s \alpha<1$ then

$$
q p_{b}(T x, T y) \leq \alpha q p_{b}(x, y)
$$

holds both for $x \in A, y \in B$ and $x \in B, y \in A$.

Fan[12] in the same paper proved Kannan contraction condition in the setting of a quasipartial b-metric space which is stated as follows:

Let $A$ and $B$ be nonempty subsets of a quasi-partial b-metric space $\left(X, q p_{b}\right)$. A cyclic mapping $T: A \cup B \rightarrow A \cup B$ is said to be a $q p_{b}$-cyclic-Kannan contraction mapping if there exists $\beta \in[0,1 / 2)$ such that if $s \geq 1, s \beta<1 / 2$ then

$$
q p_{b}(T x, T y) \leq \beta q p_{b}(x, T x)+\beta q p_{b}(y, T y)
$$

holds both for $x \in A, y \in B$ and $x \in B, y \in A$.

Inspired and motivated by the work of Fan [12], Ćirić [10] and Reich [11], in this paper we have introduced new contraction conditions for Ćirić-Reich-Rus contraction ([13]-[21]) for cyclic mappings in the setting of a quasi-partial b-metric space and discuss the existence and uniqueness of fixed points for such mappings when the underlying space is complete. Throughout this paper $N, R$ and $R^{+}$denote the set of all positive integers, set of real numbers and the set of all non-negative real numbers respectively. Here, we will recall some definition and lemma as discussed by Gupta and Gautam[9] in their paper: 


\section{Preliminaries and basic properties}

Definition 1. ([7]). A Quasi-Partial b-metric on a non-empty set $X$ is a mapping $q p_{b}$ : $X \times X \rightarrow R^{+}$such that for some real numbers $s \geq 1$ and for all $x, y, z \in X$ $\left(Q P_{b_{1}}\right) q p_{b}(x, x)=q p_{b}(x, y)=q p_{b}(y, y)$ implies $x=y$ $\left(Q P_{b_{2}}\right) \quad q p_{b}(x, x) \leq q p_{b}(x, y)$ $\left(Q P_{b_{3}}\right) \quad q p_{b}(x, x) \leq q p_{b}(y, x)$ $\left(Q P_{b_{4}}\right) \quad q p_{b}(x, y)+q p_{b}(z, z) \leq s\left[q p_{b}(x, y)+q p_{b}(y, z)\right]$

A Quasi-Partial b-metric space is a pair $\left(X, q p_{b}\right)$, where $X$ is a non-empty space and $q p_{b}$ is Quasi-Partial b-metric on $X$. The number $s$ is called coefficient of $\left(X, q p_{b}\right)$.

The following lemmas are instrumental in our result.

Lemma 1. ([9]). Let $\left(X, q p_{b}\right)$ be a Quasi-Partial b-metric space. Then the following holds (i) If $q p_{b}(x, y)=0$ then $x=y$.

(ii) If $x \neq y$ then $q p_{b}(x, y)>0$ and $q p_{b}(y, x)>0$.

Lemma 2. ([9]). let $\left(X, q p_{b}\right)$ be a Quasi-Partial b-metric space. Then (i) A sequence $\left\{x_{n}\right\} \subset X$ converges to $x \in X$ if and only if $q p_{b}(x, x)=\lim _{n \rightarrow+\infty} q p_{b}\left(x_{n}, x\right)=\lim _{n \rightarrow+\infty} q p_{b}\left(x, x_{n}\right)$ (ii) A sequence $\left\{x_{n}\right\} \subset X$ is called a cauchy sequence if and only if $\lim _{n, m \rightarrow+\infty} q p_{b}\left(x_{n}, x_{m}\right)=\lim _{m, n \rightarrow \infty} q p_{b}\left(x_{m}, x_{n}\right)$ exist and finite.

(iii) The Quasi-Partial b-metric space $\left(X, q p_{b}\right)$ is said to be complete if every sequence $\left\{x_{n}\right\} \subset X$ converges with respect to $\tau_{q p_{b}}$ to a point $x \in X$ such that $q p_{b}(x, x)=\lim _{n, m \rightarrow+\infty} q p_{b}\left(x_{n}, x_{m}\right)=\lim _{m, n \rightarrow+\infty} q p_{b}\left(x_{m}, x_{n}\right)$.

Lemma 3. ([12]). Let $\left(X, q p_{b}\right)$ be a quasi-partial b-metric space and $\left\{x_{n}\right\}_{n=1}^{\infty}$ be a sequence in $X$. If $x_{n} \rightarrow x, x_{n} \rightarrow y$ and $q p_{b}(x, x)=q p_{b}(y, y)=0$, then $x=y$.

\section{Main Result}

In this section, we extend the fixed point theorem for Ćirić-Reich-Rus contraction mappings in standard metric spaces to $q p_{b}$-cyclic-Ćirić-Reich-Rus contraction mappings in the setting of quasi-partial b-metric spaces.

Definition 2. . Let $A$ and $B$ be nonempty subsets of a quasi-partial b-metric space $\left(X, q p_{b}\right)$. A cyclic mapping $T: A \cup B \rightarrow A \cup B$ is said to be a qp $p_{b}$-cyclic-Cirić-Reich-Rus contraction mapping if there exists $\alpha \in[0,1)$ and $\beta \in[0,1 / 2)$ such that if $s \geq 1, s(\alpha+2 \beta)<1$ then

$$
q p_{b}(T x, T y) \leq \alpha q p_{b}(x, y)+\beta q p_{b}(x, T x)+\beta q p_{b}(y, T y),
$$

holds both for $x \in A, y \in B$ and $x \in B, y \in A$. 
Theorem 1. . Let $A$ and $B$ be two nonempty closed subsets of a complete quasi-partial b-metric space $\left(X, q p_{b}\right)$ and $T$ be a cyclic mapping which is a $q p_{b}$-cyclic-Cirić-Reich-Rus mapping. Then $A \cap B$ is nonempty and Thas a unique fixed point in $A \cap B$.

Proof. . Let $x \in A$, considering condition (1), we have

$$
\begin{aligned}
q p_{b}\left(T x, T^{2} x\right) & \leq \alpha q p_{b}(x, T x)+\beta q p_{b}(x, T x)+\beta q p_{b}\left(T x, T^{2} x\right) \\
(1-\beta) q p_{b}\left(T x, T^{2} x\right) & \leq(\alpha+\beta) q p_{b}(x, T x) \\
q p_{b}\left(T x, T^{2} x\right) & \leq \frac{(\alpha+\beta)}{(1-\beta)} q p_{b}(x, T x)
\end{aligned}
$$

Using (2) we get

$$
\begin{aligned}
& q p_{b}\left(T^{2} x, T x\right) \leq \alpha q p_{b}(T x, x)+\beta q p_{b}\left(T x, T^{2} x\right)+\beta q p_{b}(x, T x) \\
& q p_{b}\left(T^{2} x, T x\right) \leq \alpha q p_{b}(T x, x)+\beta\left(\frac{(\alpha+\beta)}{1-\beta}\right) q p_{b}(x, T x)+\beta q p_{b}(x, T x)
\end{aligned}
$$

Take, $\gamma=\operatorname{Max}\left\{q p_{b}(x, T x), q p_{b}(T x, x)\right\}$, then we have

$$
\begin{aligned}
& q p_{b}\left(T x, T^{2} x\right) \leq \frac{(\alpha+\beta)}{(1-\beta)} \gamma, \text { and } \\
& q p_{b}\left(T^{2} x, T x\right) \leq \frac{(\alpha+\beta)}{(1-\beta)} \gamma .
\end{aligned}
$$

Similarily,

$$
\begin{aligned}
q p_{b}\left(T^{3} x, T^{2} x\right) & \leq\left(\frac{\alpha+\beta}{1-\beta}\right)^{2} \gamma, \text { and } \\
q p_{b}\left(T^{2} x, T^{3} x\right) & \leq\left(\frac{\alpha+\beta}{1-\beta}\right)^{2} \gamma
\end{aligned}
$$

Hence,

$$
q p_{b}\left(T^{n} x, T^{n+1} x\right) \leq\left(\frac{\alpha+\beta}{1-\beta}\right)^{n} \gamma \quad \text { and } \quad q p_{b}\left(T^{n+1} x, T^{n} x\right) \leq\left(\frac{\alpha+\beta}{1-\beta}\right)^{n} \gamma
$$

for every $n \in N$.

Let $m, n \in N$ and $m<n$, using (3) and $Q P_{b_{4}}$ we obtain

$$
\begin{aligned}
& q p_{b}\left(T^{m} x, T^{n} x\right) \leq s\left[q p_{b}\left(T^{m} x, T^{m+1} x\right)+q p_{b}\left(T^{m+1} x, T^{n} x\right)\right]-q p_{b}\left(T^{m+1} x, T^{m+1} x\right) \\
& q p_{b}\left(T^{m} x, T^{n} x\right) \leq s\left[q p_{b}\left(T^{m} x, T^{m+1} x\right)+q p_{b}\left(T^{m+1} x, T^{n} x\right)\right] \\
& q p_{b}\left(T^{m} x, T^{n} x\right) \leq \operatorname{sqp}_{b}\left(T^{m} x, T^{m+1} x\right)+s^{2} q p_{b}\left(T^{m+1} x, T^{m+2} x\right)+s^{2} q p_{b}\left(T^{m+2} x, T^{n} x\right) \\
& q p_{b}\left(T^{m} x, T^{n} x\right) \leq \operatorname{sqp}_{b}\left(T^{m} x, T^{m+1} x\right)+s^{2} q p_{b}\left(T^{m+1} x, T^{m+2} x\right)+\ldots \ldots+s^{n-m} q p_{b}\left(T^{n-1} x, T^{n} x\right) .
\end{aligned}
$$


Let $\left(\frac{\alpha+\beta}{1-\beta}\right)=\delta$, then

$$
\begin{aligned}
& q p_{b}\left(T^{m} x, T^{n} x\right) \leq\left(s \delta^{m}+s^{2} \delta^{m+1}+\ldots . .+s^{n-m} \delta^{n-1}\right) \gamma \\
& q p_{b}\left(T^{m} x, T^{n} x\right) \leq s \delta^{m}\left(1+s \delta+(s \delta)^{2}+\ldots+(s \delta)^{n-m-1}\right) \gamma \\
& q p_{b}\left(T^{m} x, T^{n} x\right) \leq s \delta^{m}\left(\frac{1-(s \delta)^{n-m}}{1-s \delta}\right) \gamma
\end{aligned}
$$

Since, $s(\alpha+2 \beta)<1, s \geq 1$ then, $1-s \beta \leq 1-\beta$ and $s(\alpha+\beta)<1-s \beta$, therefore, $s\left(\frac{\alpha+\beta}{1-\beta}\right)<$ 1, i.e. $s \delta<1$. Furthermore,

$$
q p_{b}\left(T^{m} x, T^{n} x\right) \leq\left(\frac{s \delta^{m}}{1-s \delta}\right) \gamma
$$

Taking limit as $m, n \rightarrow+\infty$ in the above inequality, we have

$$
\lim _{n, m \rightarrow+\infty} q p_{b}\left(T^{m} x, T^{n} x\right) \leq 0
$$

Thus,

$$
\lim _{n, m \rightarrow+\infty} q p_{b}\left(T^{m} x, T^{n} x\right)=0
$$

Also,

$$
\begin{aligned}
& q p_{b}\left(T^{n} x, T^{m} x\right) \leq s\left[q p_{b}\left(T^{n} x, T^{m+1} x\right)+q p_{b}\left(T^{m+1} x, T^{m} x\right)\right]-q p_{b}\left(T^{m+1} x, T^{m+1} x\right) \\
& q p_{b}\left(T^{n} x, T^{m} x\right) \leq s\left[q p_{b}\left(T^{n} x, T^{m+1} x\right)+q p_{b}\left(T^{m+1} x, T^{m} x\right)\right] \\
& q p_{b}\left(T^{n} x, T^{m} x\right) \leq s^{2} q p_{b}\left(T^{n} x, T^{m+2} x\right)+s^{2} q p_{b}\left(T^{m+2} x, T^{m+1} x\right)+\operatorname{sqp}_{b}\left(T^{m+1} x, T^{m} x\right) \\
& q p_{b}\left(T^{n} x, T^{m} x\right) \leq s^{n-m} q p_{b}\left(T^{n} x, T^{n-1} x\right)+s^{n-m-1} q p_{b}\left(T^{n-1} x, T^{n-2} x\right)+\ldots \ldots+s_{b}\left(T^{m+1} x, T^{m} x\right) \\
& q p_{b}\left(T^{n} x, T^{m} x\right) \leq\left(s^{n-m} \delta^{n-1}+s^{n-m-1} \delta^{n-2}+\ldots .+s \delta^{m}\right) \gamma \\
& q p_{b}\left(T^{n} x, T^{m} x\right) \leq s \delta^{m}\left((s \delta)^{n-m-1} 1+(s \delta)^{n-m-2}+\ldots+s \delta\right) \gamma \\
& q p_{b}\left(T^{n} x, T^{m} x\right) \leq s \delta^{m}\left(\frac{1-(s \delta)^{n-m}}{1-s \delta}\right) \gamma .
\end{aligned}
$$

Taking limit as $m, n \rightarrow+\infty$ in the above inequality, we have

$$
\lim _{n, m \rightarrow+\infty} q p_{b}\left(T^{n} x, T^{m} x\right) \leq 0 .
$$

Thus,

$$
\lim _{n, m \rightarrow+\infty} q p_{b}\left(T^{n} x, T^{m} x\right)=0 .
$$

Using (4) and (5) indicate that sequence $\left\{T^{n} x\right\}_{n=0}^{+\infty}$ is a Cauchy sequence.

Since $\left(X, q p_{b}\right)$ is complete, therefore $\left\{T^{n} x\right\}_{n=0}^{+\infty}$ converges to some $u \in X$, that is,

$$
\begin{aligned}
q p_{b}(u, u) & =\lim _{n \rightarrow+\infty} q p_{b}\left(T^{n} x, u\right)=\lim _{n \rightarrow+\infty} q p_{b}\left(u, T^{n} x\right)=\lim _{n, m \rightarrow+\infty} q p_{b}\left(T^{n} x, T^{m} x\right)= \\
& =\lim _{n, m \rightarrow+\infty} q p_{b}\left(T^{m} x, T^{n} x\right)=0 .
\end{aligned}
$$


Observe that $\left\{T^{2 n} x\right\}_{n=0}^{+\infty}$ is a sequence in A and $\left\{T^{2 n-1} x\right\}_{n=1}^{+\infty}$ is a sequence in B in a way that both sequences converge to $\mathrm{u}$. Note also that $A$ and $B$ are closed, we have $u \in A \cap B$

$$
q p_{b}\left(T^{n} x, T u\right) \leq \alpha q p_{b}\left(T^{n-1} x, u\right)+\beta q p_{b}\left(T^{n-1} x, T^{n} x\right)+\beta q p_{b}(u, T u) .
$$

Taking limit as $n \rightarrow+\infty$ in the above inequality and using (6), we have

$$
\begin{aligned}
q p_{b}\left(T^{n} x, T u\right) & \leq \beta q p_{b}(u, T u) \\
\beta q p_{b}(u, T u) & \leq \beta\left[\operatorname{sqp}_{b}\left(u, T^{n} x\right)+\operatorname{sqp}_{b}\left(T^{n} x, T u\right)-q p_{b}\left(T^{n} x, T^{n} x\right)\right] \\
\beta q p_{b}(u, T u) & \leq \beta\left[\operatorname{sqp}_{b}\left(u, T^{n} x\right)+\operatorname{sqp}_{b}\left(T^{n} x, T u\right)\right] .
\end{aligned}
$$

Taking limit as $n \rightarrow+\infty$ in the above inequality, we have

$$
\beta q p_{b}(u, T u) \leq \beta \operatorname{sqp}_{b}\left(T^{n} x, T u\right) .
$$

Thus, using (7) and (8)we have

$$
q p_{b}\left(T^{n} x, T u\right) \leq \beta q p_{b}(u, T u) \leq \beta \operatorname{sqp}_{b}\left(T^{n} x, T u\right) .
$$

Since, $s \beta<1$

$$
\lim _{n \rightarrow+\infty} q p_{b}\left(T^{n} x, T u\right)=q p_{b}(u, T u)=0 .
$$

Similarly, it can be derived

$$
\lim _{n \rightarrow+\infty} q p_{b}\left(T u, T^{n} x\right)=q p_{b}(T u, u)=0 .
$$

In addition, by the contractive condition of theorem and in combination with (6) and (9), we get

$$
q p_{b}(T u, T u) \leq \alpha q p_{b}(u, u)+2 \beta q p_{b}(u, T u),
$$

so,

$$
q p_{b}(T u, T u)=0 .
$$

Equations (9),(10) and (11) show that the sequence $\left\{T^{n} x\right\}_{n=0}^{+\infty}$ to $T u$. Applying Lemma 3 , we get

$$
T u=u .
$$

For uniqueness, suppose there exist another fixed point in $A \cup B$ say w i.e. $T w=w$, by contractive condition,

$$
\begin{aligned}
& q p_{b}(u, w)=q p_{b}(T u, T w) \leq \alpha q p_{b}(u, w)+\beta q p_{b}(u, T u)+\beta q p_{b}(w, T w) \\
& q p_{b}(u, w) \leq \alpha q p_{b}(u, w)+\beta q p_{b}(u, u)+\beta q p_{b}(w, w) .
\end{aligned}
$$


Consider,

$$
\begin{aligned}
q p_{b}(u, u) & =q p_{b}(T u, T u) \\
& \leq \alpha q p_{b}(u, u)+\beta q p_{b}(u, T u)+\beta q p_{b}(u, T u) \\
& =(\alpha+2 \beta) q p_{b}(u, u) \\
(1-\alpha-2 \beta) q p_{b}(u, u) & \leq 0 .
\end{aligned}
$$

Since

$$
\alpha+2 \beta<1, \quad q p_{b}(u, u)=0 .
$$

In a similar manner,

$$
q p_{b}(w, w)=0 .
$$

Combining (12),(13) and (14) we get

$$
q p_{b}(u, w) \leq \alpha q p_{b}(u, w)
$$

Since, $\alpha \leq 1$ and $(1-\alpha) q p_{b}(u, w) \leq 0$, therefore, $q p_{b}(u, w)=0$. So we have $q p_{b}(u, u)=$ $q p_{b}(w, w)=q p_{b}(u, w)=0$, that $u=w$.

Example 1. Let $X=[0,1], A=\left[0, \frac{1}{2}\right]$ and $B=\left[0, \frac{1}{3}\right], T: A \cup B \rightarrow A \cup B$ as $T x=\frac{x}{2}$. Define the quasi partial $b$-metric as

$$
q p_{b}(x, y)=|x-y|+|x|
$$

for all $x, y \in X$. Here $\left(X, q p_{b}\right)$ is a quasi-partial b-metric space with $s=1$. We will verify that the mapping $\mathrm{T}$ is $q p_{b}$-cyclic-Ćirić-Reich-Rus contraction mapping. $T(A)=\left[0, \frac{1}{4}\right] \subset B$ and $T(B)=\left[0, \frac{1}{6}\right] \subset A$. Hence the mapping is cyclic on $X$.

Taking $\alpha=\frac{1}{2}, \beta \leq \frac{1}{2}, s(\alpha+2 \beta) \leq 1$

$$
\begin{aligned}
\frac{1}{2} q p_{b}(x, y)+\beta\left\{q p_{b}(x, T x)+q p_{b}(y, T y)\right\} & =\frac{1}{2}\{|x-y|+|x|\}+\beta\left|\frac{x}{2}\right|+|x|+\left|\frac{y}{2}\right|+|y| \geq \\
& \geq \frac{1}{2}\{|x-y|+|x|\} \geq\left\{\left|\frac{x}{2}-\frac{y}{2}\right|+\left|\frac{x}{2}\right|\right\}= \\
& =q p_{b}(T x, T y)
\end{aligned}
$$

Therefore, all conditions of Theorem 1 are satisfied and so $T$ has a fixed point (which is $0 \in A \cap B)$. 
Example 2 Let $X=\left[-\frac{\pi}{2}, \frac{\pi}{2}\right], A=\left[-\frac{\pi}{2}, 0\right]$ and $B=\left[0, \frac{\pi}{2}\right], T: A \cup B \rightarrow A \cup B$ as $T x=-\frac{\operatorname{Sin} x}{2}$. Define the quasi partial b- metric as

$$
q p_{b}(x, y)=|x-y|+|x|
$$

for all $x, y \in X$. Here $\left(X, q p_{b}\right)$ is a quasi-partial b-metric space with $s=1$. We will verify that the mapping $\mathrm{T}$ is $q p_{b}$-cyclic-Ćirić-Reich-Rus contraction mapping. $T(A)=[0,1] \subset B$ and $T(B)=[-1,0] \subset A$. Hence the mapping is cyclic on $X$.

Taking $\alpha=\frac{1}{2}, \beta \leq \frac{1}{2}, s(\alpha+2 \beta) \leq 1$

$$
\begin{aligned}
\frac{1}{2} q p_{b}(x, y)+\beta\left\{q p_{b}(x, T x)+q p_{b}(y, T y)\right\} & =\frac{1}{2}\{|x-y|+|x|\}+\beta\left|x+\frac{\sin x}{2}\right|+|x|+\left|y+\frac{\sin y}{2}\right|+|y| \geq \\
& \geq \frac{1}{2}\{|x-y|+|x|\} \geq \frac{1}{2}\{|\sin x-\sin y|+|\sin x|\} \geq \\
& \geq\left\{\left|-\frac{\sin x}{2}+\frac{\sin y}{2}\right|+\left|-\frac{\sin x}{2}\right|\right\}=q p_{b}(T x, T y)
\end{aligned}
$$

Therefore, all conditions of Theorem 1 are satisfied and so $T$ has a fixed point (which is $0 \in A \cap B)$.

Corollary 1. .Let $A$ and $B$ be two nonempty closed subsets of a complete quasi-partial bmetric space $\left(X, q p_{b}\right)$ and $T$ be a cyclic mapping which is a $q p_{b}$-cyclic-Kannan mapping. Then $A \cap B$ is nonempty and $T$ has a unique fixed point in $A \cap B$.

Proof. In theorem 1, Taking $\alpha=0$.

Corollary 2. . Let $A$ and $B$ be two nonempty closed subsets of a complete quasi-partial $b$-metric space $\left(X, q p_{b}\right)$ and $T$ be a cyclic mapping which is a $q p_{b}$-cyclic-Banach mapping. Then $A \cap B$ is nonempty and $T$ has a unique fixed point in $A \cap B$.

Proof. In theorem 1, Taking $\beta=0$.

Corollary 3. .Let $(X, d)$ is a complete metric space and $T: X \rightarrow X$ be a self-map satisfying,

$$
d(T x, T y) \leq \alpha d(x, y)+\beta d(x, T x)+\beta d(y, T y)
$$

for all $x, y \in X,(\alpha+2 \beta) \in[0,1)$. Then Thas a unique fixed point in $X$.

Proof. Since every metric space is also a quasi-partial b-metric space; with $s=1$, so as a particular case of theorem 1, if the underlying space is taken to be a metric space; sets $A=B=X$; then the corresponding corollary 3 follows. It is worth observing that the 
condition of sets $A$ and $B$ being closed is dropped in the above corollary by not assuming the space to be closed. This is justified as in the proof of the theorem, the closeness of sets $A$ and $B$ guaranteed that the fixed point thus obtained belongs to both $A$ and $B$. Since $A=B=X$, so the fixed point obtained naturally belongs to $X$.

\section{References}

[1] S. Shirali, H. L. VASudeva, Metric Spaces, Springer,(2005).

[2] I. A. BAKHTIN, The contraction mapping principle in almost metric spaces, Funct. Anal. 30 (1989) 2637.

[3] S. CzERWICK, Contraction mappings in b-metric spaces, Acta Math. Inform. Univ. Ostraviensis, 1993.

[4] S.Shukla, Partial b-metric spaces and fixed point theorems, Mediterr. J. Math., 11(2014),703-711.

[5] S. G. Matthews, Partial metric topology, general topology and its applications, Ann. N.Y. Acad. Sci. 1994;728:183-197.

[6] E.Karapinar, M. Erhan, A. Ozturk, Fixed Point theorems on Quasi-partial Metric spaces, Math.Comput.Model., 57, (2013),2442-2448.

[7] A. Gupta, P. GAUTAM, Topological Structure of Quasi-Partial b-Metric Spaces, International Journal of Pure Mathematical Sciences, (2016) 10-16.

[8] A. Gupta, P. Gautam, Some coupled fixed point theorems on Quasi-Partial b-metric spaces, International Journal of Mathematical Analysis, 9 (6)(2015), 293-306.

[9] A. Gupta, P. GAUTAM, Quasi-Partial b-metric spaces and some related fixed point theorems, Fixed point theory Appl. (18) (2015), 2-11.

[10] L. B. CIRIC, A generalization of Banach's contraction principle, Proc. Amer. Math. Soc., 45 (1974), 267-273.

[11] S. REICH, Fixed points of contractive functions , Boll. Unione Mat. Ital., 5(1972) 26-42.

[12] X. FAn, Fixed Point Theorems for Cyclic mappings in Quasi-Partial b-metric spaces, Journal of Non-linear Science and Applications,9,(2016), 2175-2189.

[13] M. R. Alfuraidan, Q. H. AnsARI, Fixed Point Theory and Graph Theory Foundation and integrative Approaches, Academic Press 2016.

[14] Malkowski, V. RakočEvić, Advanced Functional Analysis, CRS Press, Taylor and Francis Group, Boca Raton, FL,2019

[15] LJ. ĆIRIĆ, Some recent results in metrical fixed point theory, University of Belgrade, Beograd 2003, Serbia.

[16] V. TOdorČEvić, Harmonic Quasiconformal Mappings and Hyperbolic Type Metrics, Springer Nature Switzerland AG 2019

[17] L.N. Mishra, On existence and behavior of solutions to some nonlinear integral equations with applications, Ph.D. Thesis (2017), National Institute of Technology, Silchar 788 010, Assam, India. 
[18] L.N. Mishra, K. Jyoti, A. Rani, VAndana, Fixed point theorems with digital contractions image processing, Nonlinear Sci. Lett. A, Vol. 9, No.2, (2018), pp.104-115.

[19] L.N. Mishra, S.K. Tiwari, V.N. Mishra, I.A. Khan, Unique Fixed Point Theorems for Generalized Contractive Mappings in Partial Metric Spaces, Journal of Function Spaces, Volume 2015 (2015), Article ID 960827, 8 pages.

[20] L.N. Mishra, S.K. TIWARI, V.N. Mishra, Fixed point theorems for generalized weakly $S$ contractive mappings in partial metric spaces, Journal of Applied Analysis and Computation, 5 (4) (2015) 600-612.

[21] X. Liu, M. Zhou, L.N. Mishra, V.N. Mishra, B. Damjanović, Common fixed point theorem of six self-mappings in Menger spaces using $\left(C L R_{S T}\right)$ property, Open Mathematics, 16 (2018) 1423-1434. 